\title{
Comparative Analysis of Cytokine mRNA Expressions in Human Tissues with Mycobacterium tuberculosis Infection
}

Sung-Bae Park ${ }^{1,2 \S}$, Heechul Park ${ }^{1,2 \S}$, Yoon-Sung Choi ${ }^{1}$, Ji Young Park ${ }^{3}$, Dongsup Lee ${ }^{4}$, Jungho Kim ${ }^{1,2}$, Hee-Kyung Chang ${ }^{5 \dagger}$, and Sunghyun Kim ${ }^{1,2 \dagger}$

\author{
${ }^{1}$ Department of Clinical Laboratory Science, College of Health Sciences, Catholic University of Pusan, \\ Busan, Republic of Korea; \\ ${ }^{2}$ Clinical Trial Specialist Program for In Vitro Diagnostics, Brain Busan 21 Plus Program, Graduate School, \\ Catholic University of Pusan, Busan, Republic of Korea; \\ ${ }^{3}$ Department of Internal Medicine, Kosin University Gospel Hospital, Busan, Republic of Korea; \\ ${ }^{4}$ Department of Biomedical Laboratory Science, Hyejeon College, Hongsung, Republic of Korea; \\ ${ }^{5}$ Department of Pathology, Kosin University College of Medicine, Busan, Republic of Korea. \\ †Corresponding author: Sunghyun Kim, Ph. D. and Hee-Kyung Chang, M. D., Ph. D. \\ Corresponding authors: Sunghyun Kim, Ph. D. \\ Address: Department of Clinical Laboratory Science, College of Health Sciences, Catholic University of \\ Pusan, Busan, Republic of Korea, 46252 \\ Tel: $+82-51-510-0560$ \\ Fax: $+82-51-510-0568$ \\ e-mail: shkim0423@cup.ac.kr \\ Co-corresponding authors: Hee-Kyung Chang, M. D., Ph. D. \\ Address: Department of Pathology, Kosin University College of Medicine, Busan, Republic of Korea, 49267 \\ Tel: +82-10-3571-6323 \\ e-mail: changhkg@kosin.ac.kr \\ $\S$ Equal contributors: S. P and H. P contributed equally to this work.
}

\begin{abstract}
In the present study, we aimed to investigate whether an automated molecular diagnostic method based on PCR-reverse blot hybridization assay can discriminate between human Mycobacterium tuberculosis (MTB)-positive and -negative FFPE tissues and to compare the relative mRNA expression levels of various host immune markers between MTB-infected and uninfected human tissues using quantitative reverse transcription (qRT) PCR. A total of 52 human FFPE tissue samples from various regions of the body, including the lungs, lymph nodes, tendons, colon, and appendix, were collected and used for the molecular identification of Mycobacterium species and analysis of cytokine mRNA expression. As a result, IFN- $\gamma, T N F-\alpha, I P-10, C X C L 9, C X C L 11$, and GMCSF mRNA expression levels in MTB-infected tissues were significantly higher than those in uninfected samples. Additionally, the differences in the mRNA expression levels of IFN- $\gamma, C X C L 9$, and $G M-C S F$ between MTB-infected and uninfected tissues were statistically significant were statistically significant $(p<0.05)$. Correlation curve analysis indicated that the mRNA expression of IFN- $\gamma$ was inversely proportional to that of $I P-10$ and that the mRNA expression levels of $I F N-\gamma, T N F-\alpha, C X C L 9$, $C X C L 11, G M-C S F$, and TNFR were proportional and well-correlated. Furthermore, to establish marker profiles for detecting MTB infection, the statistically significant expression levels of three markers were combined. We confirmed that the combined profile of $I F N-\gamma, C X C L 9$, and GM-CSF expression levels was statistically significant $(P<0.001)$. Although the mRNA expression patterns of host immune markers may vary according to MTB infection status, these patterns may be highly correlated and can be simultaneously used as an additional indicator for diagnosing TB.
\end{abstract}



FFPE tissue.

Keywords: Tuberculosis, Mycobacterium tuberculosis, mRNA expression, Cytokine, Human

\section{Introduction}

Tuberculosis (TB) is an infectious disease caused by Mycobacterium tuberculosis (MTB) and remains a serious health problem worldwide [1]. According to a World Health Organization report in 2018, the Republic of Korea had the highest prevalence and mortality rate for TB among countries in the Organization for Economic Cooperation and Development [2]. Moreover, cases of multi-drugresistant and extensively drug-resistant TB are rapidly increasing, leading to increased difficulties in efficient TB treatment and control [3].

Several different immune markers such as cytokines, chemokines, immune regulators, and immune stimulating factors have recently been shown to play important roles in hosts infected with MTB [4]. In a study, tumor necrosis factor-alpha (TNF- $\alpha$ ), an inflammatory cytokine, was reportedly implicated in not only the proliferation and differentiation of immune cells but also in inflammatory processes, including apoptosis of MTB-infected cells [5]. In another study, interferon-gamma (IFN- $\gamma$ ) and antigen-specific T cells reportedly controlled MTB infection [6]. In addition, interleukin 2 (IL-2) levels have been found to be significantly higher in patients with active TB than in healthy control groups [7], and IL-4 has also been reported to act as an anti-inflammatory cytokine in cases of TB infection [8]. Furthermore, C-X-C motif chemokine 9 (CXCL9; a monokine induced by IFN- $\gamma$ ) levels in the plasma of patients with active pulmonary TB are higher than that in the plasma of healthy individuals [4]. CXCL10 (an IFN- $\gamma$-inducible protein of 10, IP-10) levels have also been found to be elevated in patients with TB lymphocytic pleural effusion [9], and CXCL11 (an IFN- $\gamma$ inducible T cell alpha chemoattractant) levels have been shown to be significantly increased by stimulation with an MTB antigen [10]. Importantly, research has also shown that the mRNA levels of these immune markers can be precisely measured in blood immune cells to distinguish among individuals with active TB, those with latent TB infection (LTBI), and healthy controls [11]. Granulocyte-macrophage colonystimulating factor (GM-CSF) is a biomarker that can be used to differentiate between persons with active TB and QuantiFERON TB Gold In Tube (QFT-GIT) test-positive persons [12]. Higher levels of plasma CCL11 (eotaxin) and TNF receptor (TNFR) have also been associated with loss of CD4 ${ }^{+} \mathrm{T}$ cells [13].

Here, we aimed to investigate whether an automated molecular diagnostic method based on PCR-reverse blot hybridization assay (REBA) can discriminate between human MTB-positive FFPE tissues and MTB- and NTM-negative FFPE tissues and to compare the mRNA expression levels of various host immune markers in MTB-infected human tissues using quantitative reverse transcription PCR (qRT-PCR) TaqMan probe assays.

\section{Methods}

\subsection{Patients and clinical samples}

A total of 52 human FFPE tissue samples were collected between 2011 and 2017 by the Department of Pathology of Kosin University Gospel Hospital in Busan, Republic of Korea. Clinical data indicated that five of the samples were positive and 11 were negative for MTB infection according to Ziehl-Neelsen AFB staining. The other 36 samples were not assessed using AFB staining.

\subsection{Genomic DNA extraction and Molecular identification of Mycobacterium species}

gDNA extraction from FFPE tissue samples was performed using QIAamp DNA FFPE Tissue Kit (Qiagen, Hilden, Germany) according to the manufacturer's instructions. The concentration and purity of the gDNA extracted from the FFPE tissues were measured using NanoDrop 2000 (Thermo Fisher Scientific, Waltham, MA, USA), then extracted DNA was used to confirm MTB-positive and negative using REBA Myco-ID ${ }^{\circledR}$ Kit (YD Diagnostics, Yongin, Republic of Korea) according to the manufacturer's instructions. All reactions were automatically performed by the HybREAD $480^{\circledR}$ 
System (YD Diagnostics). After the processes were complete, the data were interpreted using an HybREAD $^{\circledR} 480$ Scanner (YD Diagnostics). 


\subsection{Total RNA isolation}

Total RNA isolation from human FFPE tissue samples was performed using PureLink ${ }^{\mathrm{TM}}$ FFPE Total RNA Isolation ${ }^{\circledR}$ kit (Invitrogen, Waltham, MA, USA) according to the manufacturer's instructions. The concentration and purity of isolated total RNA from FFPE tissues were measured using NanoDrop ${ }^{\mathrm{TM}} 2000$ (Thermo Fisher Scientific), and the isolated RNA was then stored at $-80^{\circ} \mathrm{C}$ until further use.

\subsection{Reverse transcription $(\mathrm{RT})$ reaction}

RT reactions were performed using Moloney Murine Leukemia Virus Reverse Transcriptase (M-MLV RT) cDNA Synthesis kit (Thermo Fisher Scientific) and Random Hexamers (Invitrogen) according to the manufacturers' instructions. The mixture was initially heated at $65^{\circ} \mathrm{C}$ for $5 \mathrm{~min}$ and then incubated at $25^{\circ} \mathrm{C}$ for $10 \mathrm{~min}, 50^{\circ} \mathrm{C}$ for $50 \mathrm{~min}$, and $70^{\circ} \mathrm{C}$ for $15 \mathrm{~min}$. All reactions were performed using SimpliAmp Thermal Cycler (Life Technologies, Carlsbad, CA, USA). The final cDNA samples were stored at $-20^{\circ} \mathrm{C}$ until further use.

\subsection{Gene expression profiling by multiplex bead array}

Gene expression profiling for targeting the mRNA (transcript) expression levels of 25 different host immune markers, including $\mathrm{C}-\mathrm{C}$ motif chemokine ligand 2 (CCL2, monocyte chemoattractant

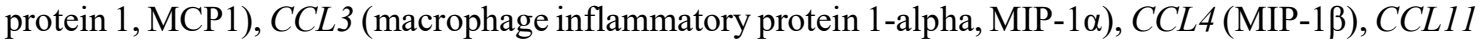
(eotaxin), C-X-C motif chemokine 8 (CXCL8, IL-8), CXCL9 (MIG), CXCL10 (IP-10), CXCL11 (ITAC), GM-CSF, IFN- $\gamma$, IFN- $\gamma$ receptor 1 (IFN- $\gamma R 1), I L-1 \alpha(\mathrm{IL}-1 \mathrm{~F} 1), I L-1 \beta$ (IL-1F2), IL-2, IL-2Ra, IL4, IL-5, IL-6, IL-6Ra, IL-10, IL-12,IL-17, IL-18,TNFRI, and TNF- $\alpha$, was analyzed using QuantiGene ${ }^{\circledR}$ Plex Gene Expression Assay (Thermo Fisher Scientific) and MAGPIX ${ }^{\circledR}$ multiplexing system (Luminex, Austin, TX, USA). All target gene expressions were normalized against three endogenous control genes encoding peptidyl-prolyl cis-trans isomerase B (PPIB), glyceraldehyde 3-phosphate dehydrogenase (GAPDH), and hypoxanthine phosphoribosyltransferase 1 (HPRT1). The process was performed according to the manufacturer's instructions.

\subsection{Quantitative RT-PCR TaqMan probe assays}

qRT-PCR TaqMan probe assays (Roche Diagnostics, Mannheim, Germany and Applied Biosystems, Waltham, MA, USA) were performed to target the mRNA expression of multiple host immune markers ; qRT-PCR primer and TaqMan probe pairs were used to amplify the mRNA of Th1type cytokines and receptors $(I F N-\gamma, T N F-\alpha, T N F R, I L-2$, and $I L-2 R)$, Th2-type cytokine (IL-4), IFN- $\gamma-$ induced chemokines (CXCL9, CXCL10, and CXCL11), chemotactic factor (CCL11), stimulating factor $(G M-C S F)$, and internal reference protein $(G A P D H)$.

\subsection{Statistical analysis}

All statistical analyses were performed using GraphPad Prism v. 5.00 (GraphPad Software, San Diego, CA, USA). Significant differences in the mRNA expression levels of MTB-positive and MTB-negative tissues, together with $90 \%$ confidence intervals (CIs), were calculated and compared using unpaired $t$-tests. Associations between the mRNA expression levels of IFN- $\gamma$ and other cytokines were measured using Spearman's rank correlation coefficient (r). An ROC curve analysis was performed to confirm clinical applicability. A $p$-value of less than 0.05 was considered statistically significant.

\section{RESULTS}

\subsection{Demographics}

The demographics of the participants are shown in Table 1. The mean age of the participants from whom samples were collected was 51.7 years (range 17-87) and the male-to-female ratio was $2.05: 1(67.3 \%$ to $32.7 \%)$. FFPE tissues were obtained from various regions of the body, including the colon $(n=14,26.9 \%)$, lungs $(n=13,25.0 \%)$, ileum $(n=7,13.6 \%)$, lymph nodes $(n=6,11.6 \%)$, skin 
( $n=3,5.8 \%)$, ears $(n=2,3.8 \%)$, and appendix, bronchus, finger joint, kidney, testis, tendon, and soft tissue (each $n=1,1.9 \%$ ).

\subsection{Mycobacterial infection pattern}

REBA Myco-ID ${ }^{\circledR}$ assay was performed to investigate mycobacterial infections; the results are shown in Table 2. Among the 52 samples, 11 samples (21\%) were MTB-positive, 20 (39\%) were NTMpositive, 8 (15\%) were MTB- and NTM-double positive, and 13 (25\%) were negative.

\subsection{Gene expression profiling of host immune markers in MTB-infected and uninfected tissue samples}

Of the 25 target genes assessed, the gene expression levels of $C X C L 9, C X C L 10, C C L 2, C C L 3$, $C C L 4, C C L 11, I L 1 \beta, I L-2 R, T N F R$, and $I L-6 R$ were higher in MTB-infected tissues than in uninfected tissues; however, the mRNA expression levels of $C X C L 9, C C L 2, C C L 3, C C L 4, I L 1 \beta$, and $I L-6 R$ varied in MTB-infected tissues, even though they exhibited higher mRNA expression levels. The mRNA expression levels of $I F N-\gamma, T N F-\alpha, I L-2, I L-4$, and $C X C L 11$ reportedly increased upon infection with MTB based on previous studies; however, the differences in the mRNA expression levels of $I L-6$ and $I L-8$ between MTB-infected and uninfected tissues were not significant (Table 3).

\section{4. mRNA expression patterns in samples according to MTB infection}

Shown in Figure 1 are the mRNA expression levels of MTB-positive and MTB-negative tissues. The mRNA expression levels of IFN- $\gamma, T N F-\alpha, C X C L 9, C X C L 10, C X C L 11$, and GM-CSF were relatively higher in MTB-positive tissues than in MTB-negative tissues. In particular, IFN- $\gamma, C X C L 9$, and $G M-C S F$ were significantly higher $(p<0.05)$ in MTB-positive tissues than in MTB-negative tissues. $I L-2$ and $C C L 11 \mathrm{mRNA}$ expression levels were lower in MTB-positive tissues than in MTBnegative tissues; however, the mRNA expression levels of $I L-2 R$ and $T N F R$ were not different between MTB-positive tissues and MTB-negative tissues. $I L-4$ mRNA expression levels were not detected owing to low levels. To establish marker profiles for detecting MTB infection, the expression levels of three markers whose differences between MTB-positive and MTB-negative tissues were statistically significant were combined and validated. The differences in the combined profile of IFN- $\gamma, C X C L 9$, and $G M-C S F$ expression levels discriminated between MTB-positive and -negative tissues and was also statistically significant $(P<0.001)$.

\subsection{Correlation curve analysis patterns of mycobacterial infection}

The correlation curve analysis was based on the expression levels of $I F N-\gamma$, which is known to specifically increase in host tissues with MTB (Figure 2). The mRNA expression levels of $I F N-\gamma$ were significantly correlated with those of $T N F-\alpha, C X C L 9, C X C L 11, G M-C S F$, and TNFR; however, there was no correlation between the mRNA expression levels of $I F N-\gamma$ and $I L-2$ or $I L-2 R$. Interestingly, the mRNA expression levels of $I F N-\gamma$ and $C X C L 10$ were inversely proportional.

\subsection{Receiver operating characteristic (ROC) curve analysis of the mRNA expression levels of host immune markers}

ROC curve analysis was performed to ensure that the results were clinically applicable (Figure 3). The area under the curve (AUC) was $0.696(p=0.0414)$ for $I F N-\gamma, 0.625(p=0.1934)$ for $T N F-\alpha$, $0.679(\mathrm{p}=0.063)$ for $C X C L 10(\mathrm{IP}-10), 0.562(\mathrm{p}=0.516)$ for $I L-2 R, 0.598(\mathrm{p}=0.312)$ for $I L-2,0.511$ $(\mathrm{p}=0.925)$ for $C C L 11,0.736(\mathrm{p}=0.015)$ for $C X C L 9,0.677(\mathrm{p}=0.068)$ for $C X C L 11,0.696(\mathrm{p}=0.044)$ for $G M-C S F$, and $0.580(\mathrm{p}=0.412)$ for TNFR. Only the $p$-values pertinent to IFN- $\gamma$ and $C X C L 9$, among 10 host immune marker genes, were significant $(p<0.05)$; the AUCs of both were approximately 0.7 . In addition, the $p$-value and the AUC of the combination of IFN- $\gamma, C X C L 9$, and GM-CSF expression levels were 0.0016 and 0.7989 , respectively.

\section{DISCUSSION}


TB remains a serious global health problem [14]. Also, NTM infections make the accurate diagnosis and treatment of TB challenging [15], and effective methods are needed to accurately identify different mycobacterial species. Therefore, we determined, using an automated molecular mycobacterial identification system, and analyzed the mRNA expression patterns of various host immune markers in tissues according to MTB infection status.

Recent studies have shown that many cytokines play an important role in cell-mediated immune responses to MTB infections [16]. So, we conducted to profile the gene expression patterns of 11 host immune markers in MTB-positive and -negative FFPE tissues.

Gene expression patterns were profiled using QuantiGene ${ }^{\circledR}$ Plex assay (Thermo Fisher Scientific), and out of a total of 25 immune cytokine genes, the mRNA expression levels of $I F N-\gamma, T N F-$ $\alpha, I L-2, I L-2 R, I L-4, C X C L 9, C X C L 10, C X C L 11$, and GM-CSF, which are known to increase upon infection with MTB, were higher than those in uninfected tissues. Specifically, TNFR and CCL11 expression levels were significantly increased. Although the expression levels of $C C L 2, C C L 3, C C L 4$, $I L 1 \beta$, and $I L-6 R$ were also significant, the differences among sample results were borderline significant and therefore were excluded.

Therefore, to definitively confirm the gene expression patterns of 11 host immune markers, we performed qRT-PCR analyses using TaqMan probes. In a previous study, $I F N-\gamma$ and $C X C L 9$ mRNA levels were found to be significantly higher in individuals with active pulmonary TB than in healthy controls [11]. In another study wherein the GM-CSF levels between IGRA-positive and -negative cases were compared, the cytokine levels in IGRA-positive cases were noted to be significantly higher than those in negative cases [13]; our results are consistent with this finding in tissue samples with and without MTB infection. Furthermore, in our ROC curve analysis, the AUCs of $I F N-\gamma, C X C L 9$, and $G M-$ $C S F$ were approximately 0.7 . To establish marker profiles that can discriminate between MTB-positive and -negative tissues, two groups, each consisting of combined and validated statistically significant mRNA levels of three immune markers, were used. A combined profile of $I F N-\gamma, C X C L 9$, and $G M-$ $C S F$ mRNA expression levels can discriminate between MTB-positive and -negative groups and was statistically significant $(P<0.001)$. With this, even when clinical molecular diagnostic assays such as PCR-REBA and nested PCR assays for detecting MTB indicate negative results, accurately diagnosing a clinical case of TB is possible by analyzing the gene expression patterns of select host immune markers. Additionally, even if DNA fragmentation occurs, the mRNA levels of cytokines remain unaffected.

Our study has some limitations. First, a small sample size was used; further research with a larger sample would provide improved significance to succeeding results. Second, the method used in this study is not easily applicable in clinical settings owing to the use of tissue samples from patients. Our analysis was conducted as a preliminary study of the gene expression patterns in peripheral wholeblood samples for the detection of TB. Further research on cytokine analysis using peripheral wholeblood samples in patients with extrapulmonary TB would be required.

\section{Conclusion}

In conclusion, the mRNA expression levels of the host immune markers $I F N-\gamma, T N F-\alpha$, CXCL9, CXCL10, CXCL11, IL-2R, and GM-CSF were higher in MTB-positive tissues than in MTBnegative tissues. Specifically, increased mRNA expression levels of $I F N-\gamma, C X C L 9$, and $G M-C S F$ were statistically significant $(p<0.05)$, and ROC curve data indicated that the AUCs of the combination of these three target mRNA expression levels exceeded 0.7. These results suggest that when TB is suspected, gene expression analysis may be used to accurately diagnose the presence of TB.

Table 1. Tissue sample sources

Characteristics $\quad n(\%)$

$\underline{\text { Age }(\text { median }=51.7 \mathrm{SD} \pm 16.3 ; \text { range }=17-87)}$

$\leq 39$

$11(21.1)$ 
40-59

$\geq 60$

$\underline{\text { Sex }}$

Male

Female

$\underline{\text { Locations }}$

Colon

Lung

Ileum

Lymph node

Skin

Ear

Bronchus

Testis

Tendon

Kidney

Appendix

Soft tissue (hand)

Finger joint
$21(40.4)$

$20(38.5)$

$35(67.3)$

$17(32.7)$

$14(26.9)$

$13(25.0)$

7 (13.6)

$6(11.6)$

$3(5.8)$

$2(3.8)$

1 (1.9)

$1(1.9)$

$1(1.9)$

$1(1.9)$

$1(1.9)$

$1(1.9)$

1 (1.9)

52 (100)

Table 2. Molecular identification of Mycobacterium species using PCR-REBA

\begin{tabular}{ccccc}
\hline $\begin{array}{c}\text { MTB infection } \\
n(\%)\end{array}$ & $\begin{array}{c}\text { NTM infection } \\
n(\%)\end{array}$ & $\begin{array}{c}\text { MTB \& NTM infection } \\
n(\%)\end{array}$ & $\begin{array}{c}\text { Negative } \\
n(\%)\end{array}$ & $\begin{array}{c}\text { Total } \\
n(\%)\end{array}$ \\
\hline $11(21)$ & $20(39)$ & $8(15)$ & $13(25)$ & $52(100)$ \\
\hline
\end{tabular}

Table 3. mRNA expression levels of host immune markers measured using gene expression profiling assay

\begin{tabular}{ll} 
Target gene & $\begin{array}{l}\text { Relative median fluorescent intensity (MFI) } \\
\text { value (range) }\end{array}$ \\
\hline$I F N-\gamma$ & $3.25(0-6.4)$ \\
$I N F G R I$ & $1.75(0-4.4)$ \\
$T N F-\alpha$ & $3.4(0-4.6)$ \\
$T N F R S F 1 A$ & $21.9(2.1-29.1)$ \\
$I L-1 A$ & $2.3(0-5.2)$ \\
$I L-1 \beta$ & $4.45(0-14.4)$ \\
$I L-2$ & $2.3(0.8-7.5)$ \\
$I L 2 R A$ & $4.75(0.7-8.9)$ \\
$I L-4$ & $2.65(0-4.2)$ \\
$I L-5$ & $1.8(0-2.5)$ \\
$I L-6$ & $0.7(0-1.1)$ \\
$I L-6 R$ & $7.4(1.0-27.1)$ \\
$I L-8$ & $0.3(0.1-0.5)$ \\
$I L-10$ & $2.75(0.6-4.7)$ \\
$I L-12 A$ & $3.35(0-5.8)$ \\
$I L-17 A$ & $2.1(0.7-3.5)$ \\
$I L-18$ & $2.65(0.2-4.6)$ \\
$C X C L 9$ & $1.5(0.1-24.8)$
\end{tabular}




\begin{tabular}{ll} 
CXCL10 (IP-10) & $4.4(1.1-9.8)$ \\
CXCL11 & $2.1(1-2.8)$ \\
$C C L 2$ & $2.9(0.6-44)$ \\
$C C L 3$ & $4.85(1.7-29.6)$ \\
$C C L 4$ & $5.05(0-22.4)$ \\
$C C L 11$ & $10.45(3.9-13)$ \\
$C S F 2$ & $2.95(1.4-3.9)$ \\
\hline
\end{tabular}



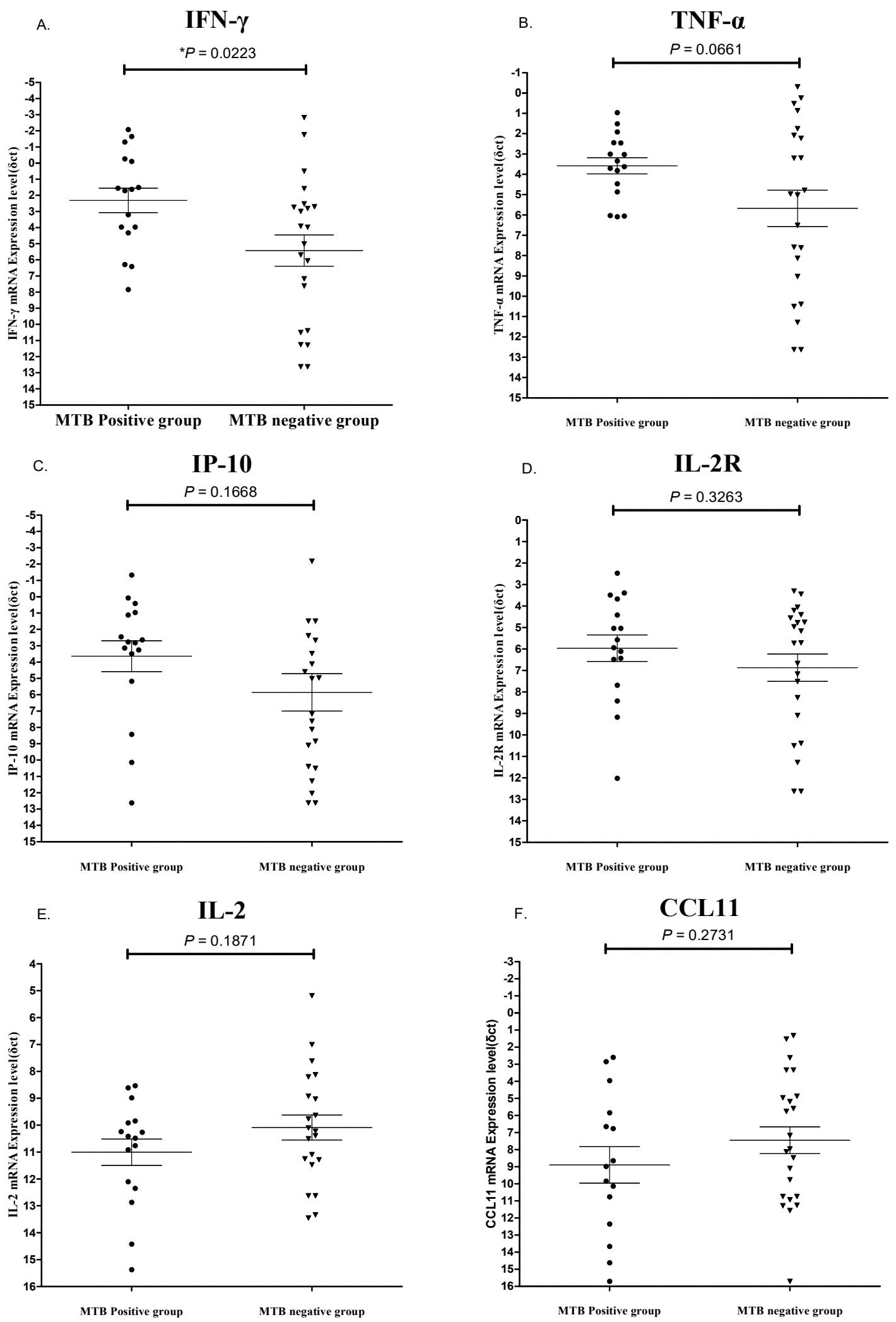

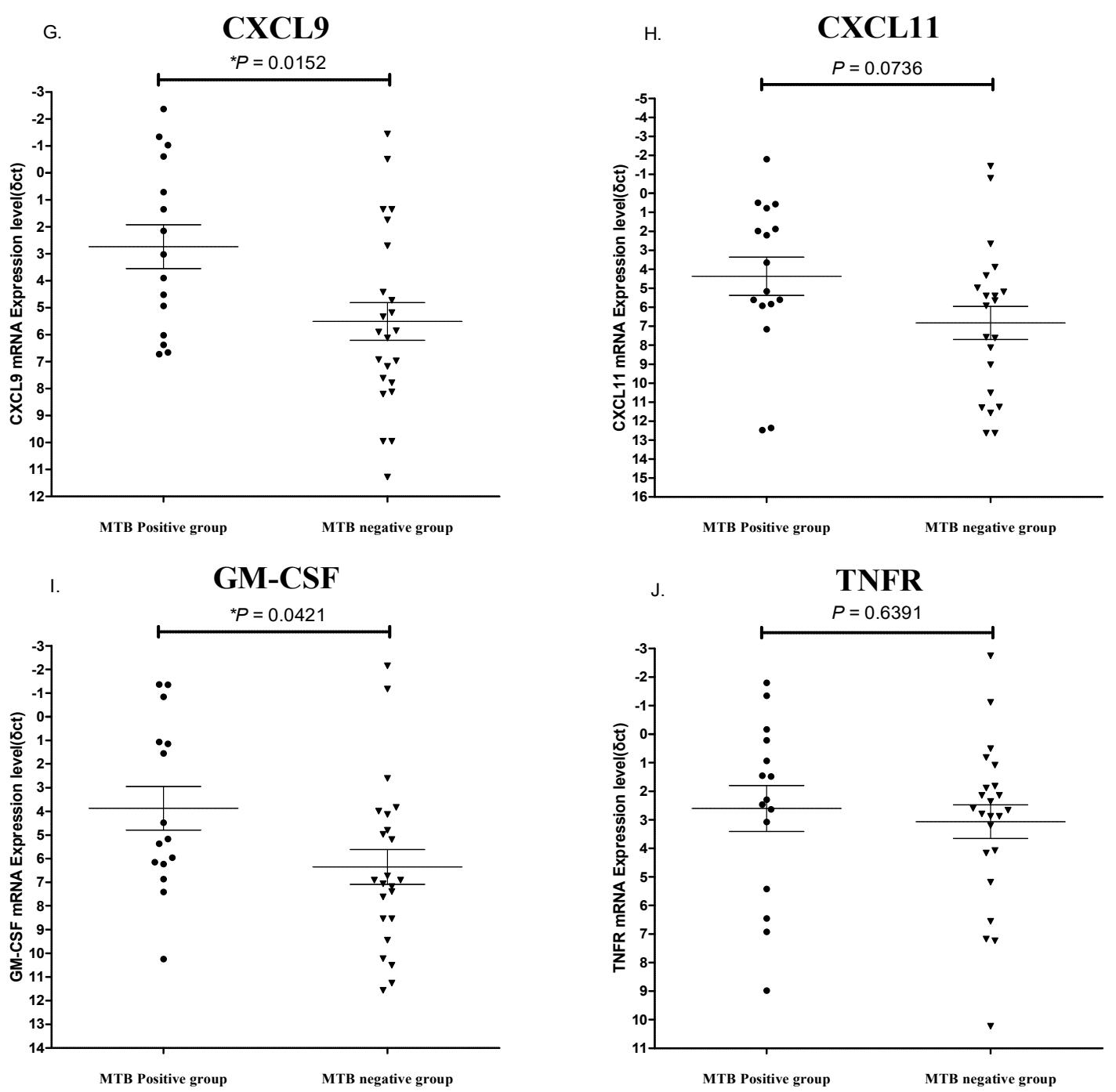

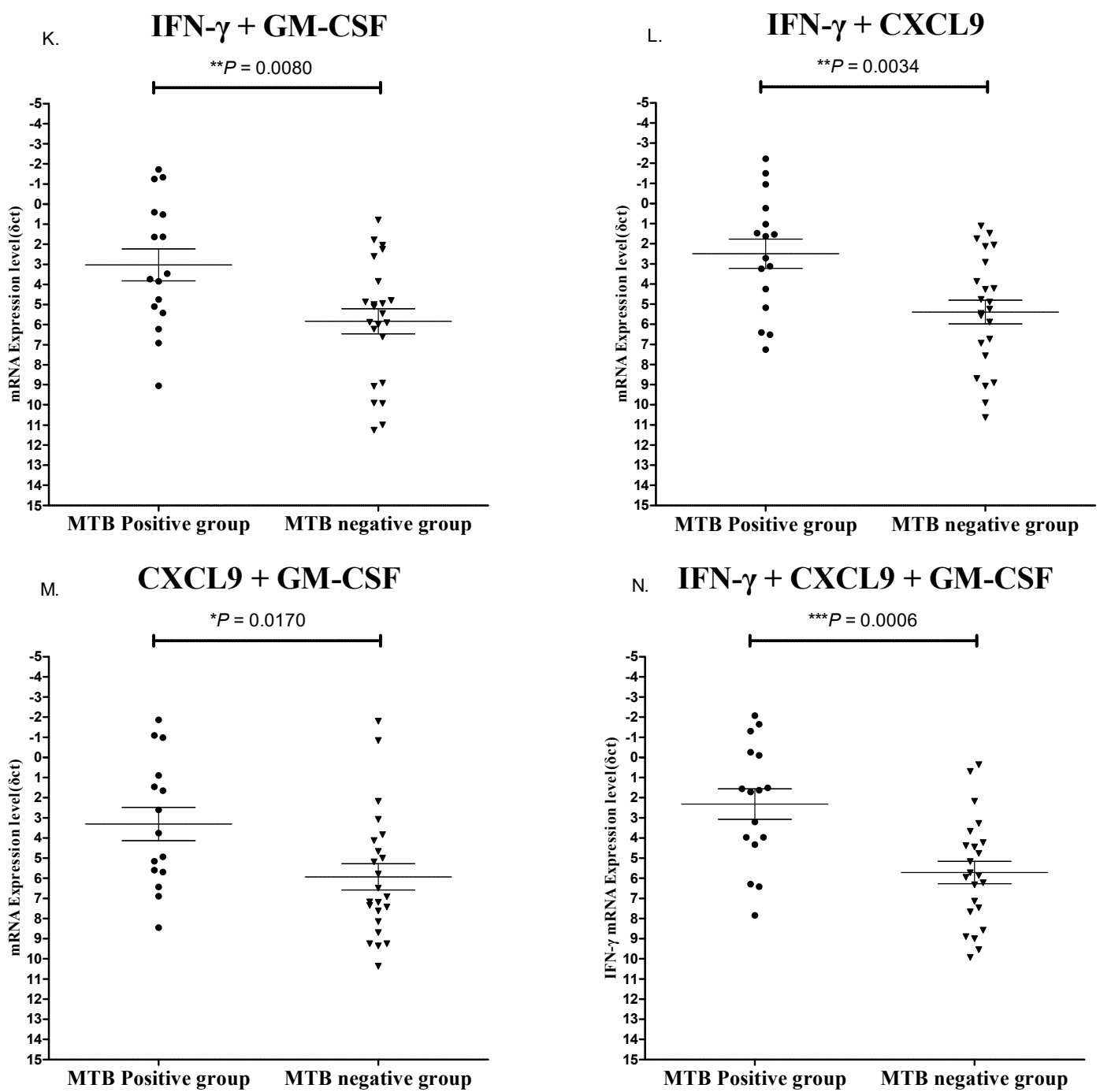

Figure 1. Comparison of the relative mRNA expression levels of host immune markers in tissues. A. Interferon-gamma $(I F N-\gamma)$, B. Tumor necrosis factor-alpha $(T N F-\alpha)$. C. Interferon gamma-inducible protein $10(I P-10)$. D. Interleukin-2 receptor $(I L-2 R)$. E. $I L-2$. F. CCL 11 (Eotaxin). G. Chemokine (CX-C motif) ligand 9 (CXCL9). H. CXCL11. I. Granulocyte-macrophage colony-stimulating factor (GM$C S F)$. J. TNFR. K. IFN- $\gamma+G M-C S F$. L. IFN- $\gamma+C X C L 9$. M. CXCL9 + GM-CSF. N. IFN- $\gamma+C X C L 9$ $+G M-C S F$. 

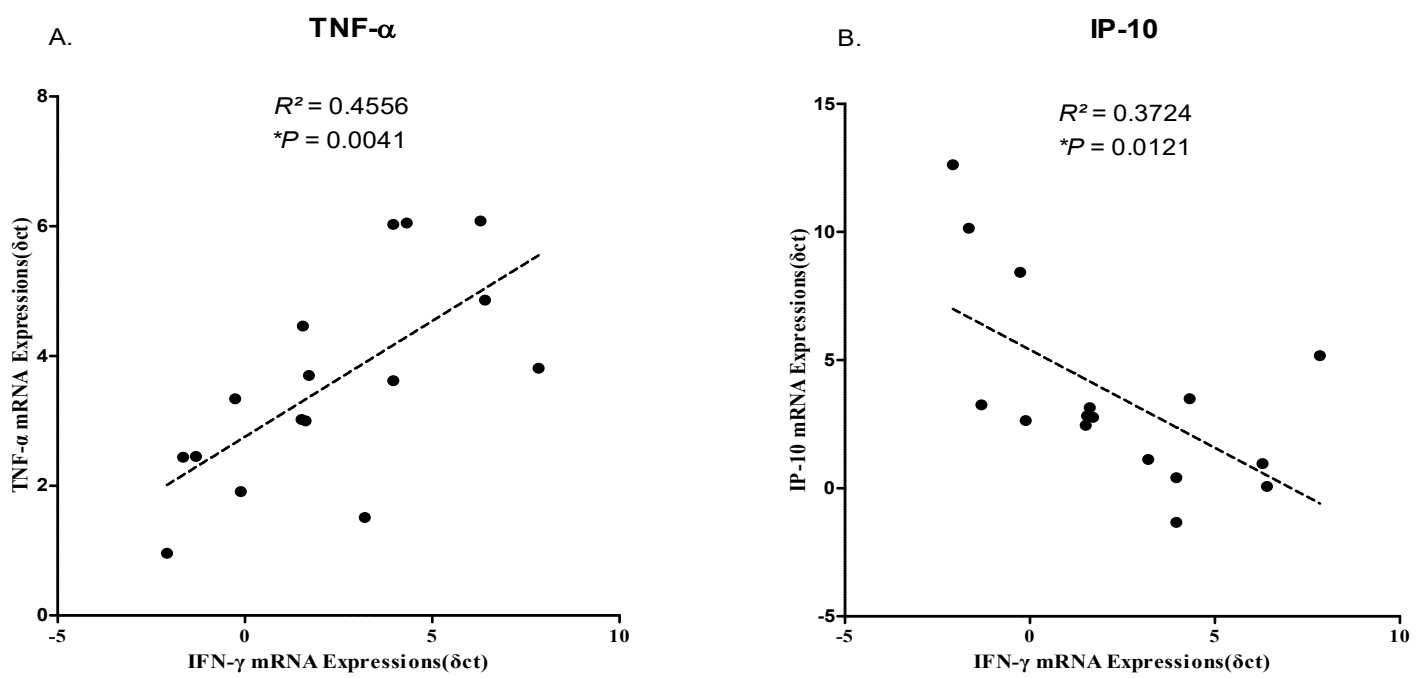

c.

\section{IL-2R}

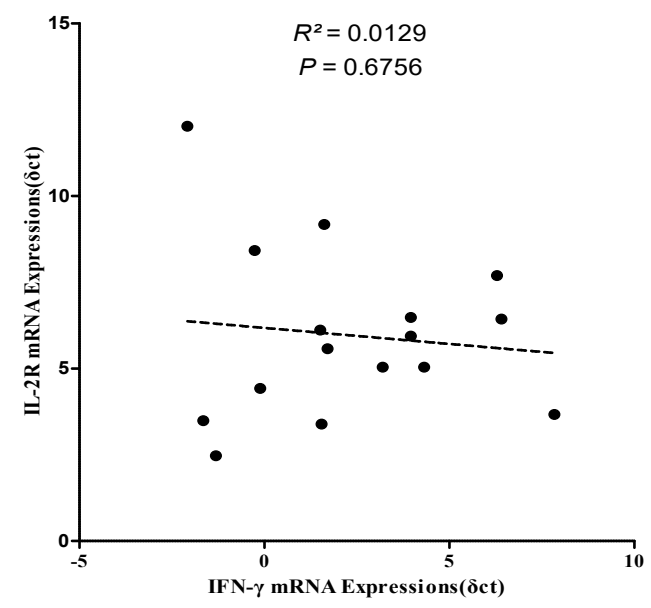

D.

IL-2

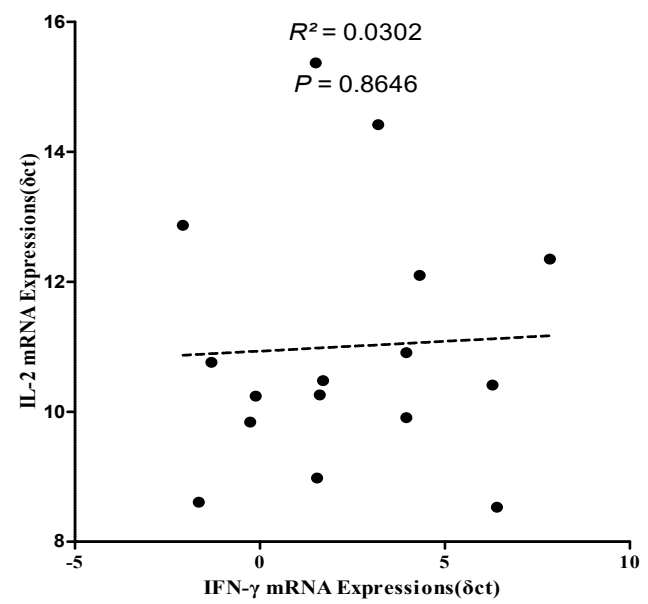

E. CCL11
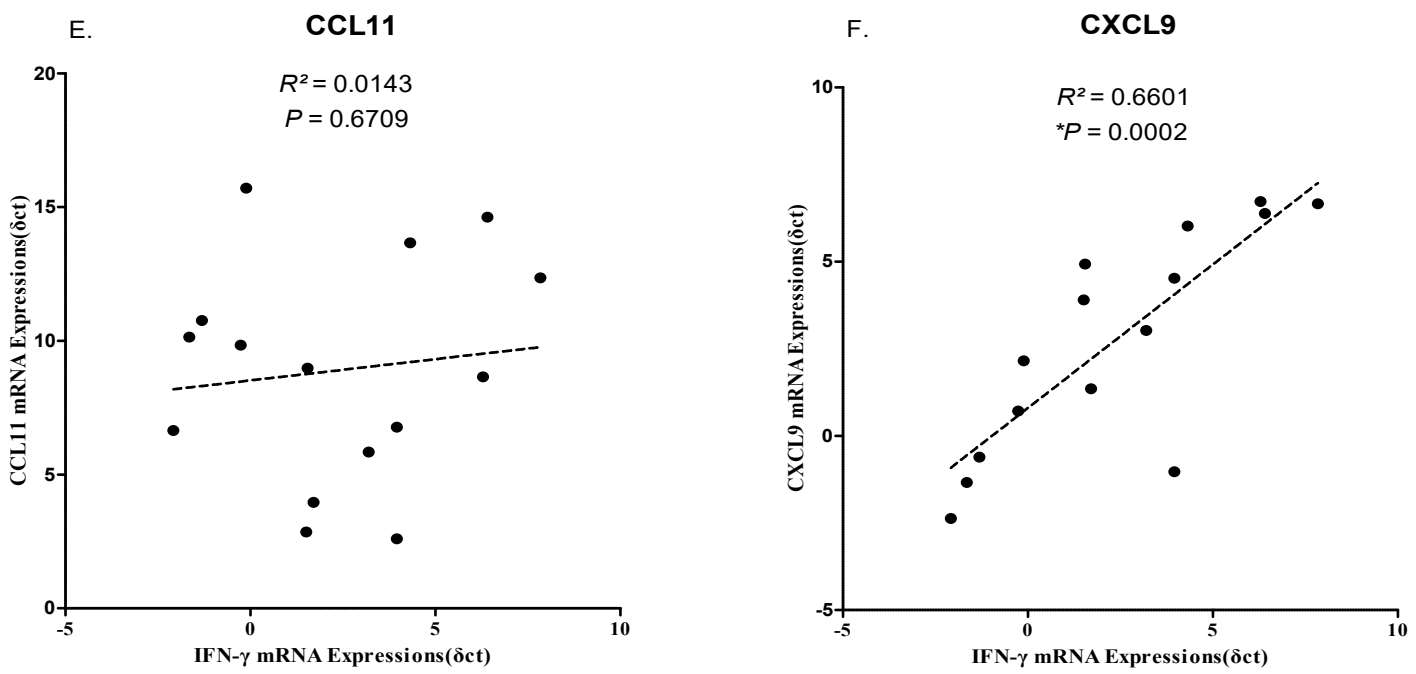
G.

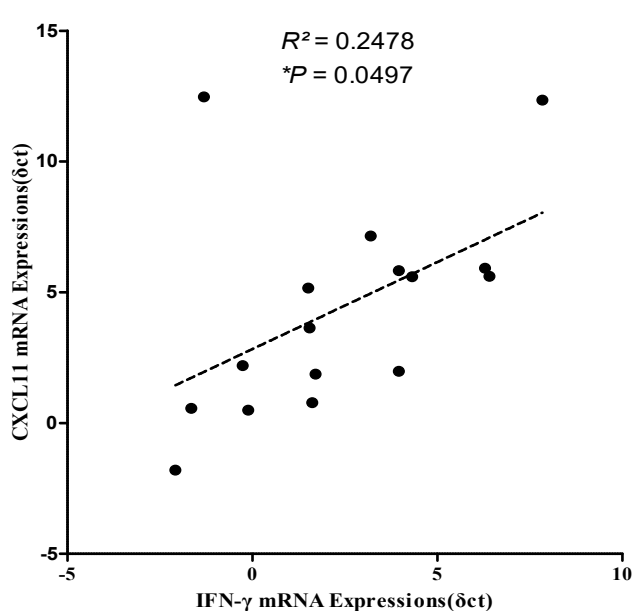

I.

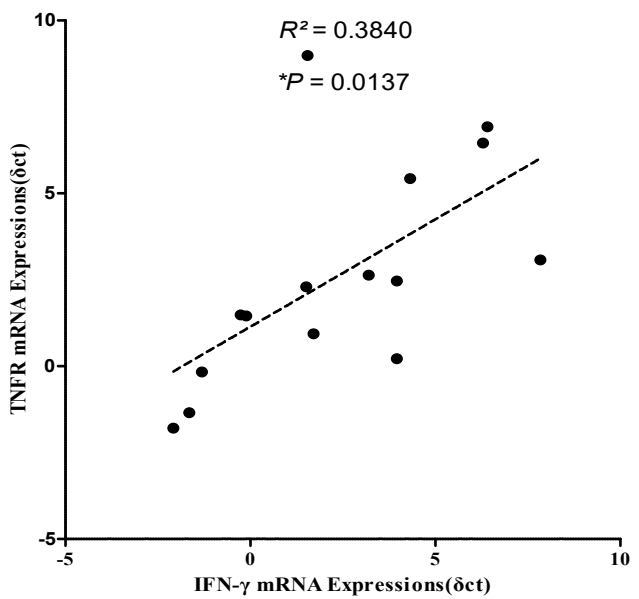

H.

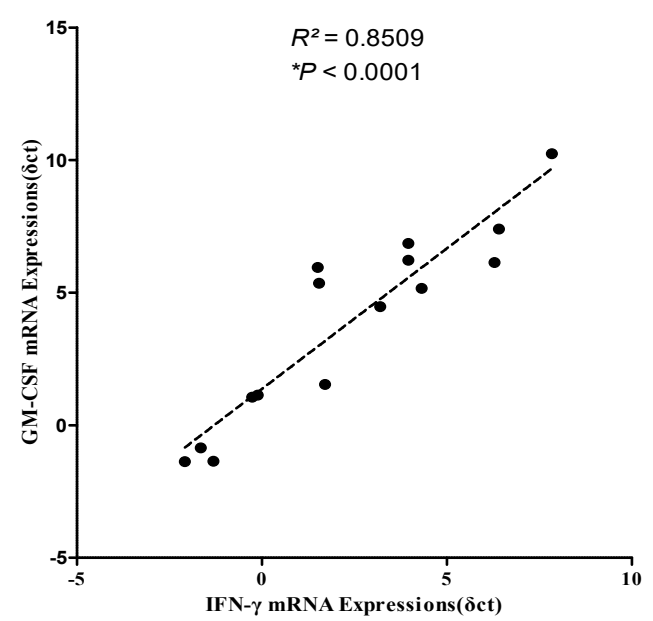

Figure 2. Correlation curve analysis of mRNA expression levels of IFN- $\gamma$ and other host immune markers in tissues. A. Tumor necrosis factor-alpha $(T N F-\alpha)$. B. Interferon gammainducible protein 10 (IP-10). C. Interleukin-2 receptor $(I L-2 R)$. D. IL-2. E. CCL11 (Eotaxin). F. Chemokine (C-X-C motif) ligand 9 (CXCL9). G. CXCL11. H. Granulocyte-macrophage colonystimulating factor $(G M-C S F)$. I. TNFR. 

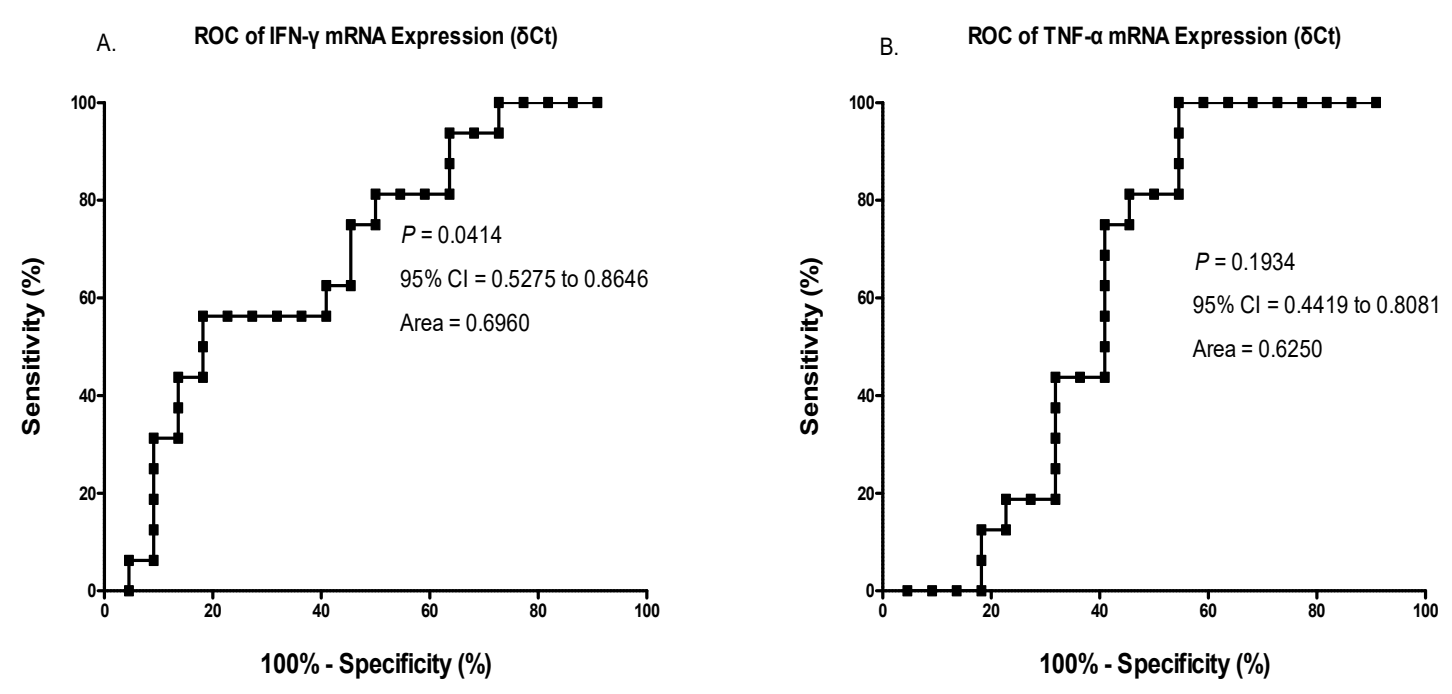

C. ROC of IP-10 mRNA Expression (סCt)

D. ROC of IL-2R mRNA Expression (סCt)
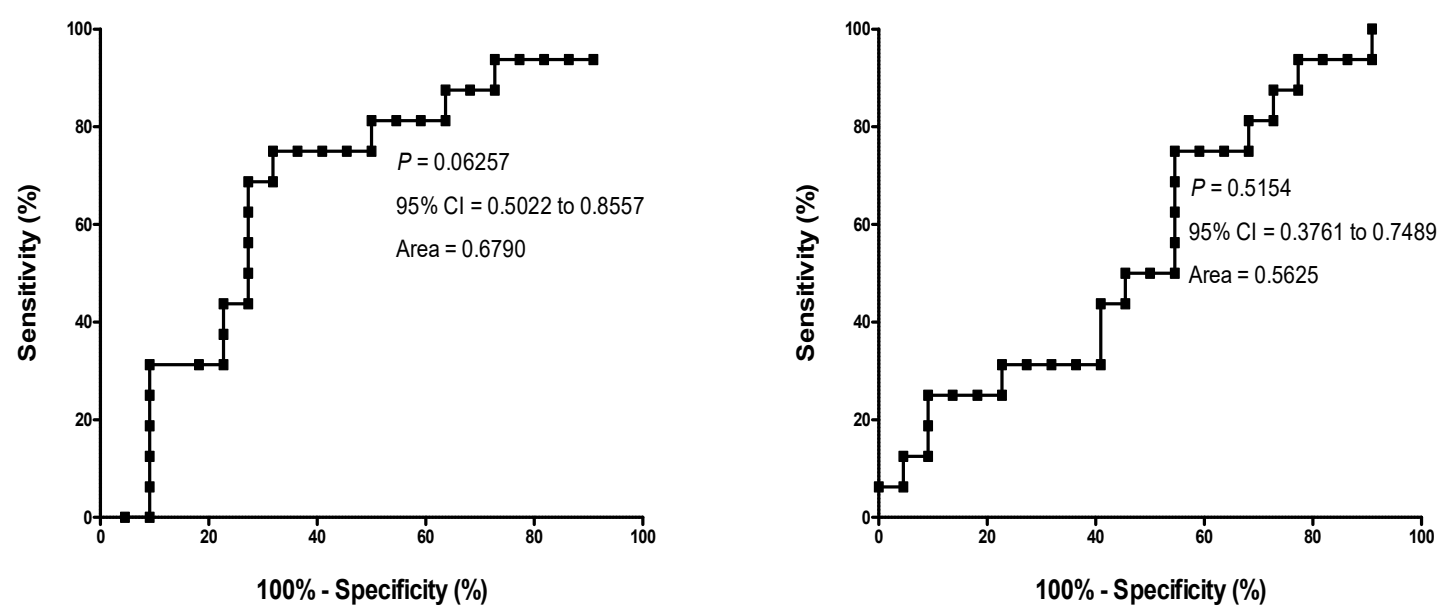

E. ROC of IL-2 mRNA Expression ( $\delta C t)$
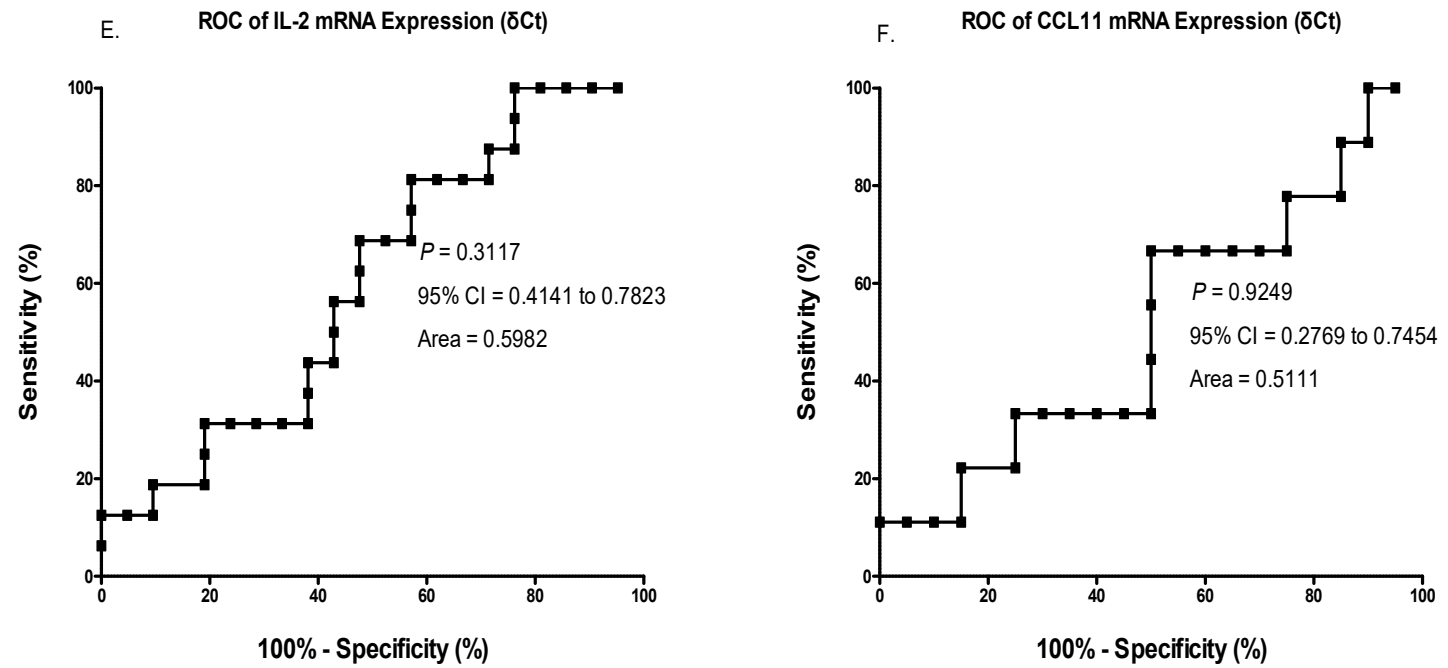

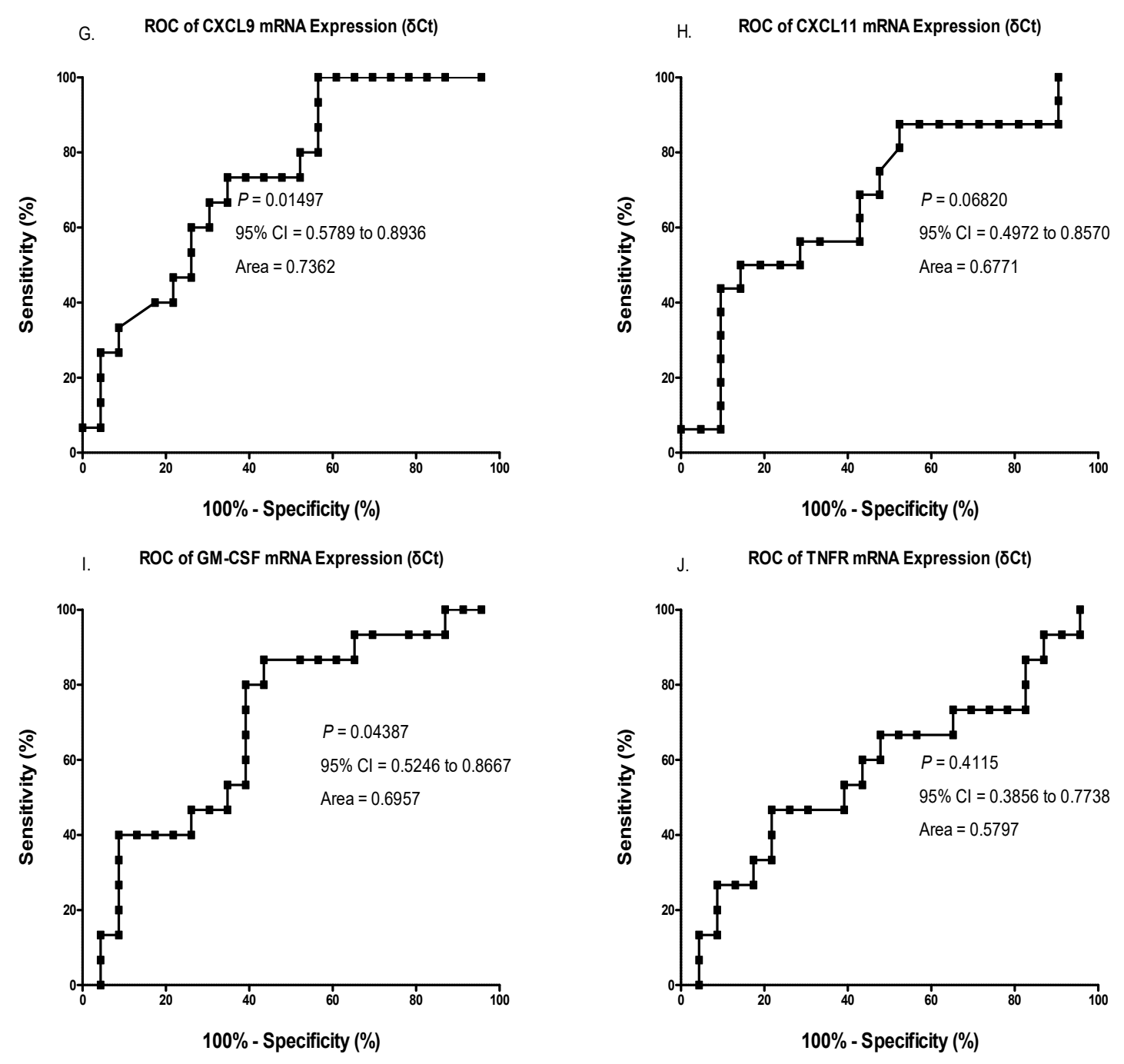

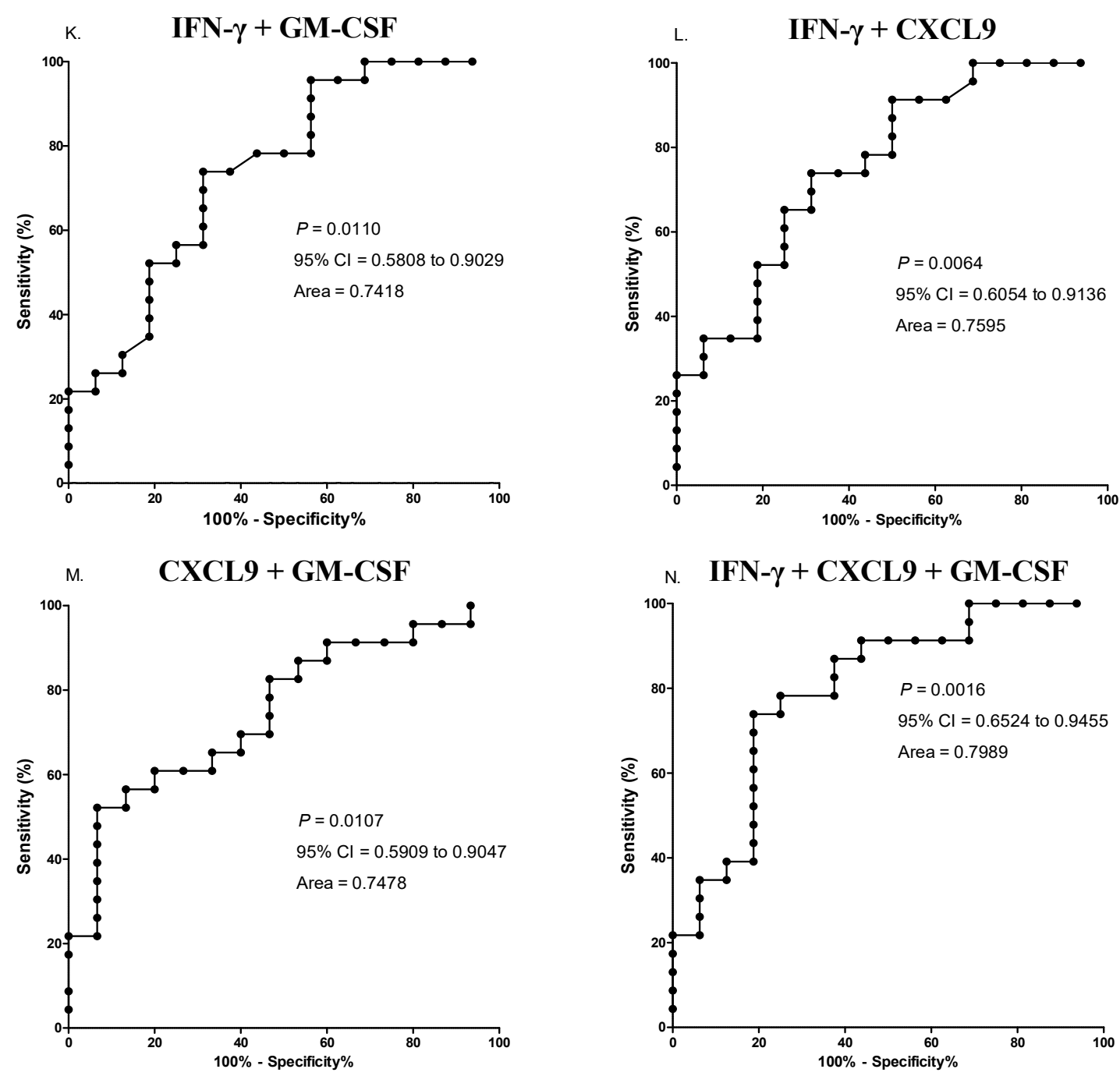

Figure 3. Receiver operating characteristic (ROC) curve analysis of host immune marker mRNA expression levels in tissues. A. Interferon-gamma $(I F N-\gamma)$, B. Tumor necrosis factor-alpha $(T N F-\alpha)$. C. Interferon gamma-inducible protein $10(I P-10)$. D. Interleukin-2 receptor $(I L-2 R)$. E. IL-2. F. CCL11 (Eotaxin). G. Chemokine (C-X-C motif) ligand 9 (CXCL9). H. CXCL11. I. Granulocyte-macrophage colony-stimulating factor $(G M-C S F)$. J. TNFR. K. IFN- $\gamma+G M-C S F$. L. IFN- $\gamma+C X C L 9$. M. CXCL9 $+G M-C S F$. N. IFN- $\gamma+C X C L 9+G M-C S F$.

\section{Author Contributions:}

Conceptualization, H.C., S.K.; Design and analyzed, S.P., H.P.; methodology, J.P., Y.C.; review and editing, J.K., D.L. All authors read and approved the final paper.

Acknowledgement: This study was supported by Basic Science Research Program through the National Research Foundation of Korea (NRF) funded by the Ministry of Education (NRF2016R1C1B108888 and 2020R1C1007169) and Brain Busan 21 Plus project.

Funding: This research did not receive any specific grant from funding agencies in the public, commercial, or not for profit sectors. 
Institutional Review Board Statement: This study was approved by the Institutional Review Board of the Kosin University Gospel Hospital (KUGH 2017-11-042).

Informed Consent Statement: FFPE tissue samples were collected after obtaining informed consent for research with the approval of the Biobank of Kosin University Gospel Hospital. All experiments were performed in accordance with relevant guidelines and regulations.

\section{Data Availability Statement:}

The data analyzed during this study are included in this paper. Some of the datasets are available from the corresponding author upon reasonable request.

Conflicts of Interest: The authors declare no conflict of interest.

\section{References}

1. Kaur G, Chander AM, Kaur G, Maurya SK, Nadeem S, Kochhar R, et al. A genomic analysis of Mycobacterium immunogenum strain CD11_6 and its potential role in the activation of T cells against Mycobacterium tuberculosis. BMC Microbiol. 2019, 19, 64. doi:10.1186/s12866-0191421-y.

2. World Health Organization. 2018. Global tuberculosis report.

3. Park YS, Lee CH, Lee SM, Yang SC, Yoo CG, Kim YW, et al. Rapid increase of non-tuberculous mycobacterial lung diseases at a tertiary referral hospital in South Korea. Int J Tuberc Lung Dis. 2010, 14, 8. 1069-1071.

4. Kim S, Lee H, Kim H, Kim Y, Cho J, Jin H, et al. Diagnostic performance of a cytokine and IFN$\gamma$ induced chemokine mRNA assay after Mycobacterium tuberculosis-specific antigen stimulation in whole blood from infected individuals. J Mol Diagn. 2015, 17, 1. 90-99. doi:10.1016/j.jmoldx.2014.08.005.

5. Keane J, Balcewicz-Sablinska M, Remold H, Chupp G, Meek B, Fenton M, et al. Infection by Mycobacterium tuberculosis promotes human alveolar macrophage apoptosis. Infect Immun. 1997, 65, 1. 298-304. doi:10.1128/IAI.65.1.298-304.1997.

6. Mogues T, Goodrich M, Ryan L, LaCourse R, North R. The relative importance of T cell subsets in immunity and immunopathology of airborne Mycobacterium tuberculosis infection in mice. $J$ Exp Med. 2001, 193, 3. 271-280. doi:10.1084/jem.193.3.271.

7. Sun Q, Wei W, Sha W. Potential role for Mycobacterium tuberculosis specific IL-2 and IFN- $\gamma$ responses in discriminating between latent infection and active disease after long-term stimulation. PLoS One. 2016, 11, 12. e0166501. Doi:10.1371/journal.pone.0166501.

8. Qi H, Sun L, Jin YQ, Shen C, Chu P, Wang S, et al. rs2243268 and rs2243274 of Interleukin-4 (IL-4) gene are associated with reduced risk for extrapulmonary and severe tuberculosis in Chinese Han children. Infect Genet Evol. 2014, 23. 121-128. doi:10.1016/j.meegid.2014.01.031.

9. Santos A, Correa R, Ribeiro-Alves M, Soares A, Mafort T, Leung J, et al. 2018. Application of Venn's diagram in the diagnosis of pleural tuberculosis using IFN- $\gamma$, IP-10 and adenosin deaminase. PLoS One. 2018, 13, 8. e0202481. doi:10.1371/journal.pone.0202481.

10. Chung W, Lee K, Jung Y, Kim Y, Park J, Sheen S, et al. Serum CXCR3 ligands as biomarkers for the diagnosis and treatment monitoring of tuberculosis. Int J Tuberc Lung Dis. 2015, 19, 12. 1476-1484. doi:10.5588/ijtld.15.0325.

11. Jeong Y, Hur Y, Lee H, Kim S, Cho J, Chang J, et al. Discrimination between active and latent tuberculosis based on ratio of antigen-specific to mitogen-induced IP-10 production. J Clin Microbiol. 2015, 53, 2. 504-510. doi:10.1128/JCM.02758-14.

12. Balcells M, Ruiz-Tagle C, Tiznado C, Garcia P, Naves R. Diagnostic performance of GM-CSF and IL-2 in response to long-term specific-antigen cell stimulation in patients with active and 
latent tuberculosis infection. Tuberculosis (Edinb.). 2018, 112, 110-119. doi:10.1016/j.tube.2018.08.006.

13. Gutierrez-Rivas M, Jimenez-Sousa MA, Rallon N, Jimenez JL, Restrepo C, Leon A, et al. High plasma levels of sTNF-R1 and CCL11 are related to CD4+ T-cells fall in human immunodeficiency virus elite controllers with a sustained virologic control, Front Immunol. 2018, 18, 9. 1399. doi:10.3389/fimmu.2018.01399.

14. Lee HJ, Ko HJ, Kim S, Jung YJ. Pasakbumin A control the growth of Mycobacterium tuberculosis by enhancing the autophagy and production of antibacterial mediators in mouse macrophages. PLoS One. 2019, 14, 3. e0199799. doi:10.1371/journal.pone.0199799.

15. Nasiri M, Dabiri H, Fooladi A, Amini S, Hamzehloo G, Feizabadi M. High rates of nontuberculous mycobacteria isolation from patients with presumptive tuberculosis in Iran. New Microbes New Infect. 2017, 6, 21. 12-17. doi:10.1016/j.nmni.2017.08.008.

16. Frahm M, Goswami ND, Owzar K, Hecker E, Mosher A, Cadogan E, et al. Discriminating between latent and active tuberculosis with multiple biomarker responses. Tuberculosis (Edinb). 2011, 91, 3. 250-256. doi:10.1016/j.tube.2011.02.006. 